\title{
Corela
}

Cognition, représentation, langage

16-1 | 2018

Vol. $16, \mathrm{n}^{\circ} 1$

\section{La représentation du terrorisme dans le discours du Parlement européen (2014-2017)}

Rachele Raus

\section{OpenEdition}

Journals

Édition électronique

URL : http://journals.openedition.org/corela/6423

DOI : $10.4000 /$ corela.6423

ISSN : $1638-573 \mathrm{X}$

Éditeur

Cercle linguistique du Centre et de I'Ouest - CerLICO

Référence électronique

Rachele Raus, «La représentation du terrorisme dans le discours du Parlement européen (2014-2017) », Corela [En ligne], 16-1 | 2018, mis en ligne le 12 juillet 2018, consulté le 19 avril 2019. URL : http:// journals.openedition.org/corela/6423; DOI : 10.4000/corela.6423

Ce document a été généré automatiquement le 19 avril 2019

\section{(c) (i) (3) (2)}

Corela - cognition, représentation, langage est mis à disposition selon les termes de la licence Creative Commons Attribution - Pas d'Utilisation Commerciale - Partage dans les Mêmes Conditions 4.0 International. 


\title{
La représentation du terrorisme dans le discours du Parlement européen (2014-2017)
}

\author{
Rachele Raus
}

\section{Introduction ${ }^{1}$}

1 Plusieurs études (Oger, Ollivier-Yanniv 2006; Krieg-Planque, Oger 2010) ont désormais montré la tendance des discours institutionnels à opérer un « lissage » de la matérialité discursive. Ces procédés finiraient par aboutir à la réalisation d'un discours monolithique et homogène où toute trace de polyphonie disparaîtrait dans un discours «instituant » (Oger, Ollivier-Yanniv 2003). Dans des conditions de production similaire, où l'ordre du discours tendrait à figer la matérialité discursive dans des configurations stables, bref un «style formulaire » (Cussò, Gobin 2008: 9) caractérisé par la présence de véritables « routines» (Née, Sitri, Véniard 2016: 72-76), qu'en est-il des mots ${ }^{2}$ et surtout des référents auxquels ils renvoient par et dans le discours?

2 Pour répondre à cette question, nous avons décidé d'analyser la manière dont le terrorisme est représenté dans le discours du Parlement européen (désormais PE). Il s'agira de voir par une analyse lexicale, qui sera à la fois quantitative et qualitative, comment le terrorisme finit par évoquer un référent discursif précis, qui est lié aux conditions de production du discours parlementaire et à la présence de l'« eurojargon » (Raus 2013a) beaucoup plus qu'à la présence d'«imaginaires socio-discursifs » (Charaudeau 2007) ${ }^{3}$ provenant des discours politiques nationaux.

Cela dit, nous verrons également la manière dont les discours politiques nationaux peuvent encore être présents et laisser leur traces dans la polyphonie qui caractérise, malgré tout, le discours institutionnel du PE. De cette manière, nous montrerons que le référent en question découle plutôt d'un compromis entre la représentation contractuelle figée par l'eurojargon communautaire ${ }^{4}$ et la tentative d'en fournir une représentation qui s'adapte mieux au positionnement national français. 
4 Après avoir présenté le corpus et la méthode d'analyse adoptée, nous analyserons le lexique, tout d'abord avec une approche purement quantitative (paragraphe 2), et ensuite avec une approche qualitative, ce qui nous permettra à la fois d'observer l'utilisation argumentative des mots qui traitent du terrorisme (paragraphe 3) et de retracer les marques discursives de la présence d'un positionnement énonciatif précis par rapport à l'objet du discours concerné (paragraphe 4). Tout cela nous amènera à répondre, dans les conclusions, à la question que nous avons posée au tout début à propos de la représentation du terrorisme en tant que référent discursif.

\section{Le corpus et la méthode d'analyse}

5 La huitième législature du Parlement européen, qui commence en 2014 et qui est actuellement en cours, nous a permis de rassembler un corpus de données relativement récent et homogène. Dans chaque législature, en effet, le PE décide de la structure des documents à produire et des procédures qui les concernent et dont les caractéristiques sont décrites et normalisées dans le règlement parlementaire. Notre corpus se compose des rapports d'initiative et législatifs du Parlement européen qui ont été produits à partir de cette législature et qui sont disponibles en langue française jusqu'en décembre 2017. Notre analyse portera sur les versions finales des documents concernés mais, là où cela s'est avéré nécessaire, nous avons également pris en compte les amendements qu'ils ont subis lors de leur rédaction.

6 En tant que genre discursif «routinier» (Maingueneau 2007: 30), les rapports parlementaires ont des spécificités lexicales, discursives et textuelles précises que nous avons décrites ailleurs (Raus 2017 : 96) et dont nous nous limitons ici à rapporter les caractéristiques principales, notamment le fait que ces documents sont constitutivement multilingues, ce qui permet aux traducteurs de doubler les instances énonciatives premières, en corédigeant la version finale de ces textes (Raus 2013b : 57, 84-85). Ajoutons que les conditions de production (Charaudeau, Maingueneau $2002: 118$ ) de ces documents se caractérisent par des prescriptions qui prévoient, entre autres, le respect des recommandations contenues dans les manuels de rédaction, l'utilisation de termes recommandés, qui sont publiés dans la base de données terminologique multilingue IATE ${ }^{5}$ , et l'utilisation de mémoires de traduction qui tendent à figer la matérialité discursive dans des dits préfabriqués. Ces conditions matérielles finissent par favoriser l'émergence d'un discours institutionnel redevable de la présence d'« éléments de langage " prescrits (Krieg-Planque, Oger 2017), qui produiraient un lissage énonciatif et une écriture formalisée, voire standardisée, capable d'opérer, entre autres, la dépersonnification maximale du discours (par exemple, par la présence massive de la non-personne ou par la routinisation du discours) et un "flou» conceptuel (Krieg-Planque 2012: 155) qui caractérise surtout les termes utilisés en discours. Lors de l'aboutissement du rapport parlementaire final, ce lissage, qui est également le résultat d'une énonciation collective négociée, tend à aplatir la dimension constitutivement polyphonique ${ }^{6} \mathrm{du}$ discours parlementaire, cette dernière restant, en revanche, visible dans les amendements proposés pendant la discussion du projet de rapport.

7 À ce sujet, nous avons voulu analyser la manière dont le terrorisme est représenté dans les rapports parlementaires qui constituent notre corpus afin de comprendre jusqu'à quel point la présence du flou conceptuel et du lissage peuvent contribuer à déterminer la représentation de ce référent discursif. Pour ce faire, nous avons déchargé les versions 
françaises des rapports disponibles dans le site du PE qui contiennent les mots " terrorisme » et «terroriste», ce qui nous a permis de décharger 13 textes au total. De ces documents, 11 sont des rapports d'initiative et 2 sont des rapports législatifs concernant la proposition de directives. Nous avons pu ainsi constater qu'aucun de ces documents n'a été produit par la Commission sur le terrorisme, une commission parlementaire qui a été créée au début de la législation en cours et qui, en fait, ne semble pas encore avoir commencé ses travaux. La plupart des documents déchargés du site du $\mathrm{PE}$ ont été produits par la Commission des affaires étrangères. Une partie résiduelle du corpus (3 documents) est le fruit de l'initiative de la Commission des Libertés civiles, de la justice et des affaires intérieures et un seul document a été proposé par la Commission des affaires constitutionnelles.

Signalons qu'à ce corpus de travail nous avons ajouté un corpus de référence plus large, qui est constitué par deux résolutions du PE ayant affaire au terrorisme et par des fiches terminologiques provenant de la base de données IATE que l'Union européenne met à disposition des traducteurs.

Quant à la méthode d'analyse, nous avons tout d'abord procédé à l'analyse quantitative des mots qui ont directement affaire au terrorisme ("terrorisme » et " terroriste ») à l'aide du logiciel de lexicométrie AntConc et du logiciel textométrique Tropes. Nous avons volontairement utilisé ces deux outils, qui se sont révélés fort complémentaires pour résoudre des questions liées à la prétendue " transparence du discours » (Née $2017: 163$ ). La comparaison des résultats et leur contextualisation ont permis en effet de mieux orienter notre analyse et nos questionnements.

Une fois obtenus les premiers résultats par la démarche quantitative, nous avons également analysé l'utilisation discursive des mots concernés par rapport à l'argumentation et par rapport aux positionnements des énonciateurs concernés. Pour ce faire, nous avons eu recours aux outils d'analyse traditionnellement mis à disposition par la lexicologie discursive (Mortureux 1997) et par l'analyse du discours «à la française » (Dufour, Rosier 2012:5).

\section{L'analyse quantitative à l'aide des outils de traitement automatique du texte}

11 Bien que nous ayons défini comme "quantitative " l'analyse à l'aide de logiciels de traitement automatique des textes, nous tenons à préciser que même lors de cette étape, l'analyse reste en fait «hybride », au sens où elle tient également compte de l'approche qualitative. En effet, l'observation des configurations lexicales et discursives ne peut pas être exempte d'une part, de l'analyse contextuelle des segments retracés et de l'autre, d'un questionnement des données qui ne se limite pas à la seule prise en compte des données chiffrées. C'est d'ailleurs la raison pour laquelle nous avons utilisé deux logiciels, l'un de lexicométrie (AntConc) et l'autre de textométrie (Tropes), qui nous ont permis de mieux comprendre et expliquer les résultats obtenus par l'analyse quantitative, d'autant plus que si le premier privilégie les simples occurrences lexicales, le deuxième permet d'observer les thèmes qui sont liés à ces occurrences et qui sont triés par « univers de références » (i.e. les domaines ou champs lexicaux). documents, mais nous a également suggéré des pistes de recherche sur les utilisations 
discursives des mots, étant donné que ces logiciels ont permis de récupérer les configurations d'énoncés ayant affaire directement aux mots ou aux thèmes concernés.

Par rapport aux résultats quantitatifs, nous avons rapporté dans le tableau 1 les fréquences et les cooccurrences majeures des mots « terrorisme » et « terroriste » d'après le logiciel AntConc:

\begin{tabular}{|l|l|l|}
\hline Mot & Fréquence & Configurations lexicales (syntagmes) fréquentes \\
\hline Terrorisme & 252 occurrences & $\begin{array}{l}\text { Lutte contre le (98 occurrences) / } \\
\text { Lutter contre le (14 occurrences) + terrorisme } \\
\text { Actes de (22 occurrences) }\end{array}$ \\
\hline Terroriste & 79 occurrences & $\begin{array}{l}\text { Radicalisation (16 occurrences) + terroriste } \\
\text { Menace (13 occurrences) } \\
\text { Propagande (12 occurrences) }\end{array}$ \\
\hline Terroristes & 287 occurrences & $\begin{array}{l}\text { Organisations (74 occurrences) } \\
\text { Groupes (17 occurrences) + terroristes } \\
\text { Actes (11 occurrences) } \\
\text { Attaques (9 occurrences) }\end{array}$ \\
\hline
\end{tabular}

Tableau 1 : Fréquences et cooccurrences de « terrorisme » et « terroriste » (source : logiciel AntConc )

Précisons que "terroriste/s » renvoie surtout à l'adjectif et très rarement au substantif. En effet, l'analyse quantitative montre que le substantif, utilisé au pluriel, est présent 6 fois dans l'expression « recrutement de/s terroristes » et uniquement dans le rapport de Rachida Dati. Au sujet des acteurs, l'absence presque totale du substantif montre qu'on privilégie des "catégories floues", notamment des « organisations / groupes" (et plus rarement « réseaux » / « filières ») terroristes, ce qui permet d'éviter de parler de vrais « sujets " (Perrot 2002: 211). Cette attitude semble trouver une confirmation indirecte dans la base de données terminologique de l'Union européenne IATE, où l'on peut noter d'une part l'absence du terme juridique " terroriste ${ }^{8}$ et de l'autre la présence d'une fiche concernant l'entrée « organisation terroriste ».

Par rapport aux configurations lexicales, remarquons la présence de plusieurs syntagmes. La plupart d'entre eux sont également enregistrés dans IATE (par exemple: "acte, attaque, groupe, infraction, menace, organisation" + "terroriste ») à l'exception de " lutte contre le terrorisme ": en effet, cette dernière expression n'est présente que dans des appellations (ISO 2009 : 36-37), l'entrée privilégiée pour renvoyer au substantif étant plutôt «lutte antiterroriste $»^{9}$. Cela nous permet de faire un tout premier constat découlant du fait que le préfixe " anti- » régit normalement un substantif ou un adjectif. Dans le cas de "terroriste", nous avons une parfaite équivalence des deux et, par conséquent, dans l'expression "lutte antiterroriste", "antiterroriste» pourrait être considéré comme adjectif qualifiant la lutte comme antiterroriste mais pourrait 
également laisser entendre la périphrase « lutte contre les terroristes ", avec le substantif qui renvoie à l'actant. Or, la reformulation « lutte contre le terrorisme ", forme nominale qui, par ailleurs, est plus fréquente que l'équivalent verbal « lutter contre le terrorisme ", permet de privilégier encore une fois le style abstrait, dépourvu de tout actant, qui caractérise les discours institutionnels (Nugara $2011: 46$ ), notamment les discours qui ont une valeur juridique (les rapports législatives) ou qui s'y rapprochent, tout en gardant encore une valeur politique (les rapports d'initiative).

Ces remarques nous permettent de confirmer la tendance du PE à opter pour un discours qui est caractérisé par l'abstraction et par l'utilisation d'un style nominal, ce qui renvoie également à la présence de l'« eurojargon » communautaire ${ }^{10}$.

Notons enfin que dans le tableau 1, l'expression « prévention du terrorisme » est absente ${ }^{11}$. En effet, le mot "prévention» est utilisé surtout en cooccurrence avec la « radicalisation » (le syntagme " prévention de la radicalisation » est utilisé 38 fois dans le corpus) et, en tant que confirmation supplémentaire de la présence d'un style nominal, nous remarquons qu'il est plus fréquent par rapport au syntagme verbal «prévenir la radicalisation » (10 occurrences). C'est le logiciel Tropes qui nous a permis d'ajouter ces réflexions sur la prévention, vu que la radicalisation figure parmi les thèmes liés au terrorisme et que la radicalisation est liée justement au thème de la prévention.

La recherche menée par Tropes confirme généralement les données d'AntConc, comme on peut le voir dans les tableaux 2 et 3 :

\begin{tabular}{|c|c|c|}
\hline Thèmes à gauche & Thème & Thèmes à droite \\
\hline $\begin{array}{l}\text { Délit (163 occurrences) } \\
\text { (« infraction-s, violation, } \\
\text { crime...») }\end{array}$ & \multirow{4}{*}{$\begin{array}{l}\text { Terrorisme } \\
\text { («terrorisme, terroriste-s, } \\
\text { antiterrorisme ») }\end{array}$} & $\begin{array}{l}\text { Délit (111 occurrences) } \\
\text { ( « infraction-s, violation, crime, vols, } \\
\text { criminels, traffic, piratage... ») }\end{array}$ \\
\hline $\begin{array}{l}\text { Combat (123 occurrences) } \\
\text { (« lutte, attaque, combat, } \\
\text { affrontement...») }\end{array}$ & & $\begin{array}{l}\text { Combat (6 occurrences) } \\
(\text { («lutte, attaque, combat, } \\
\text { affrontement...») }\end{array}$ \\
\hline $\begin{array}{l}\text { Organisation } \\
\text { occurrences) } \\
(\text { (« organisation-s... })\end{array}$ & & \\
\hline $\begin{array}{l}\text { Insécurité (19 occurrences) } \\
(« \text { menace/ risque ») }\end{array}$ & & \\
\hline
\end{tabular}

Tableau 2 : Le thème « terrorisme » par rapport aux autres (source : logiciel Tropes)

\begin{tabular}{|l|l|l|}
\hline Thèmes à gauche & Mot & Thèmes à droite \\
\hline Combat (116 occurrences) & & Radicalisation (8 occurrences) \\
\cline { 1 - 1 } Acte (27 occurrences) & Terrorisme & Combat (4 occurrences) \\
& &
\end{tabular}




\begin{tabular}{|c|c|c|}
\hline Radicalisation (8 occurrences) & & \\
\hline Infraction (154 occurrences) & \multirow{5}{*}{ Terroriste-s } & Infraction (86 occurrences) \\
\hline Organisation (74 occurrences) & & Islamisme (4 occurrences) \\
\hline Groupe (18 occurrences) & & \\
\hline Radicalisation (17 occurrences) & & \\
\hline Propagande (12 occurrences) & & \\
\hline
\end{tabular}

Tableau 3 : Les mots « terrorisme » et « terroriste-s » par rapport aux thèmes (source : logiciel Tropes)

Par la recherche sur des mots précis (tableau 3), Tropes permet en outre de confirmer que si «terrorisme » privilégie des cooccurrences à gauche qui concernent le domaine du «combat» (c'est l'expression «lutte contre» d'AntConc), l'adjectif «terroriste-s » s'accompagne à gauche de mots concernant le domaine "infraction" (l'expression « infraction-s terroriste-s » d'AntConc).

Par l'analyse des thèmes, on peut avancer l'hypothèse que la représentation du terrorisme qui découle des tableaux 2 et 3 est celle d'un objet problématique et menaçant, lié à des groupes qui agissent de manière criminelle et auxquels il faut s'opposer. Remarquons que, contrairement à ce que l'on pourrait imaginer, cette représentation n'est pas liée à la composante religieuse, l'islamisme n'étant évoqué que 4 fois et étant absent dans les données d'AntConc. À cet égard, la radicalisation, qui pourrait évoquer l'élément religieux, est en fait un concept plus large, comme le précise la députée Rachida Dati dans son rapport de 2015 : « la radicalisation ne doit pas être associée à une idéologie ou à une religion particulière, mais (...) elle peut toucher n'importe quelle idéologie ou religion".

Cela dit, les thèmes présents nous permettent d'ajouter des éléments d'analyse supplémentaires, notamment dès lors que nous constatons la présence massive du thème « délit » en cooccurrence avec les mots du terrorisme concernés (tableau 2). Un détour par IATE nous permet d'affirmer qu'en effet la plupart des mots utilisés en cooccurrences avec « terrorisme » et « terroriste » dans le discours européen sont en fait des termes qui appartiennent au domaine du droit pénal : ainsi en va-t-il pour « acte, attaque, groupe, infraction, menace, organisation ». Par conséquent, au-delà de la représentation que les mots du terrorisme utilisés pourraient évoquer pour un public de non-experts, la représentation que les parlementaires en fournissent est celle d'un objet abstrait (la fiche d'IATE précise d'ailleurs qu'«il n'existe pas de définition du terrorisme universellement reconnue »), qui valide tout de même des sanctions et des réactions précises vis-à-vis de groupes et d'organisations "terroristes ». C'est justement en ce sens que, à l'aide de la démarche qualitative, nous allons voir de plus près comment cet objet est construit pour valider des pratiques. 


\section{L'analyse qualitative du corpus}

\subsection{Du mot-argument au processus de lissage énonciatif}

L'analyse qualitative montre que le terrorisme devient le prétexte à la validation de pratiques variées, avant tout la prévention, dans une configuration qui rappelle la rhétorique de la guerre à la terreur (war on terror) américaine. À ce sujet, nous citons ces quelques citations tirées de notre corpus :

La radicalisation des citoyens de l'Union, qui peut aller jusqu'à leur départ au combat auprès d'organisations terroristes telles que l'État islamique, constitue une réelle menace sécuritaire pour l'Union européenne, ses États membres et ses voisins. Face à l'accélération de ce phénomène, les mesures de répression ne suffisent plus. L'Union européenne se doit d'adopter une nouvelle stratégie, fondée davantage sur la prévention. Ce rapport mettra donc l'accent sur la mise en place d'une attitude proactive en matière de prévention de la radicalisation et $\mathrm{du}$ recrutement de citoyens de l'Union par des organisations terroristes.

Rapporteure : Rachida Dati (exposé des motifs ; 03/11/2015)

La lutte contre le terrorisme exige une stratégie globale regroupant un grand nombre de domaines d'action différents, parmi lesquels figurent la prévention, la lutte contre la radicalisation et la déradicalisation. Le Parlement européen et le Conseil ont récemment, à plusieurs occasions, souligné la nécessité de cette approche globale dans la lutte contre le terrorisme.

Rapporteure : Monika Hohlmeier (exposé des motifs ; 12/07/2016)

$\mathrm{Au}$ cours des dernières années, criminalité et terrorisme n'ont cessé d'évoluer. Ils ont gagné en audace, en sophistication et, de plus en plus, transcendent les frontières. Et vu ce qu'il en coûte, tout indique que, de plus en plus, les citoyens attendent de l'Union européenne qu'elle prenne des mesures plus drastiques pour combattre le crime organisé et le terrorisme.

C'est dans cet esprit que le " programme de Stockholm » invitait la Commission à présenter une proposition concernant l'utilisation des données PNR pour la prévention et la détection des infractions terroristes et des infractions graves (...) Rapporteur : Timothy Kirkhope (exposé des motifs ; 07/09/2015)

Dans ces extraits, le terrorisme, ressenti comme menace, sert à légitimer des pratiques de prévention de la radicalisation ou autre soit par la modalité déontique (l'obligation morale "se doit» aussi bien que la nécessité «exige») soit par la modalisation en discours second (« les citoyens attendent de l'Union qu'elle prenne des mesures»), deux stratégies qui naturalisent le discours, et, par conséquent, l'action envisagée.

De cette manière, le terrorisme devient un véritable «mot-argument » (Mortureux 2007) qui facilite l'adhésion aux pratiques souhaitées sans recourir à d'autres assertions. Il n'est donc pas étonnant qu'il figure également comme mot-argument dans le seul document de notre corpus qui soit produit par la Commission des affaires constitutionnelles et qui concerne la volonté de l'Union de se doter d'une nouvelle structure :

[le PE] juge nécessaire, compte tenu de l'intensité de la menace terroriste, de renforcer les capacités de l'Union en matière de lutte contre le terrorisme et la criminalité organisée internationale; souligne qu'outre le renforcement de la coordination entre les autorités et agences compétentes dans les États membres, Europol et Eurojust doivent obtenir de véritables compétences et capacités d'enquête et de poursuites (...) note que ce n'est qu'en renforçant la politique étrangère et de sécurité commune que l'Union européenne peut apporter des solutions réalistes aux défis et menaces émergents en matière de sécurité, et ainsi lutter contre le terrorisme et restaurer la paix, la stabilité et l'ordre dans les pays 
de son voisinage ;

Rapporteur : Guy Verhofstadt (20/12/2016) port de Rachida Dati, dont nous rapportons quelques-uns des amendements proposés (à droite dans le tableau 4), amendements qui ne seront pas approuvés à la fin de la procédure :

\section{Amendement 14 \\ Kristina Winberg}

A. considérant que plus de 5000 citoyens européens ont rejoint des organisations terroristes et notamment les rangs de Daech en Iraq et en Syrie
A. considérant que plus de 5000 extrémistes religieux européens ont rejoint des rangs de Daech en Iraq et en Syrie organisations terroristes et notamment les 


\begin{tabular}{|c|c|}
\hline \multicolumn{2}{|l|}{$\begin{array}{l}\text { Amendement } 15 \\
\text { Franz Obermayr }\end{array}$} \\
\hline $\begin{array}{l}\text { A. considérant que plus de } 5000 \text { citoyens } \\
\text { européens ont rejoint des organisations } \\
\text { terroristes et notamment les rangs de Daech } \\
\text { en Iraq et en Syrie }\end{array}$ & $\begin{array}{l}\text { A. considérant que plus de } 5000 \text { citoyens } \\
\text { européens ont rejoint des organisations } \\
\text { terroristes islamistes et notamment les } \\
\text { rangs de Daech en Iraq et en Syrie }\end{array}$ \\
\hline \multicolumn{2}{|l|}{$\begin{array}{l}\text { Amendement } 16 \\
\text { Vicky Maeijer }\end{array}$} \\
\hline $\begin{array}{l}\text { A. considérant que plus de } 5000 \text { citoyens } \\
\text { européens ont rejoint des organisations } \\
\text { terroristes et notamment les rangs de Daech en } \\
\text { Iraq et en Syrie }\end{array}$ & $\begin{array}{l}\text { A. considérant que plus de } 5000 \text { djihadistes } \\
\text { européens ont rejoint des organisations } \\
\text { terroristes et notamment les rangs de Daech } \\
\text { en Iraq et en Syrie }\end{array}$ \\
\hline \multicolumn{2}{|l|}{$\begin{array}{l}\text { Amendement } 33 \\
\text { Franz Obermayr }\end{array}$} \\
\hline $\begin{array}{l}\text { B. considérant que les attaques terroristes à } \\
\text { Paris, à Copenhague et à Tunis de ce début } \\
\text { d'année } 2015\end{array}$ & $\begin{array}{l}\text { B. considérant que les attaques terroristes } \\
\text { islamistes à Paris, à Copenhague et à Tunis } \\
\text { de ce début d'année } 2015\end{array}$ \\
\hline \multicolumn{2}{|l|}{$\begin{array}{l}\text { Amendement } 138 \\
\text { Kristina Winberg }\end{array}$} \\
\hline $\begin{array}{l}\text { 2. invite la Commission européenne à établir } \\
\text { une définition commune de la notion de } \\
\text { «combattants étrangers" et à entreprendre } \\
\text { une étude exhaustive du processus et des } \\
\text { différentes influences qui conduisent à la } \\
\text { radicalisation }\end{array}$ & $\begin{array}{l}\text { 2. invite la Commission européenne à établir } \\
\text { une définition commune de la notion de } \\
\text { djihadistes et à entreprendre une étude } \\
\text { exhaustive du processus et des différentes } \\
\text { influences qui conduisent à la radicalisation }\end{array}$ \\
\hline
\end{tabular}

Tableau 4 : Les amendements au rapport de Rachida Dati du 3 novembre 2015

29 Les amendements proposés qui insistent sur l'élément religieux (« extrémistes religieux / islamistes / djihadistes ») ne seront pas retenus dans la version finale du rapport, qui sera alors le produit d'un vrai et propre lissage de l'hétérogénéité diaphasique. Un mécanisme similaire caractérise le rapport de Joëlle Bergeron du 10 mai 2017, où les mots « djihad » et « djihadiste » des amendements ne sont pas retenus dans la version finale du document.

Nous pouvons donc affirmer que la représentation du terrorisme est celle d'une catégorie menaçante relativement floue, qui s'incarne dans des groupes et des organisations criminelles variées et qui permet de déclencher des ripostes variées, voire proactives, de la part de l'Union européenne. Parmi ces groupes, nous remarquons la présence, somme toute rare, d'Al Quaïda (3 occurrences) et du Front al-Nosra (3 occurrences), et surtout celle de « Daech », dont l'utilisation discursive mérite une analyse ponctuelle à part. 


\subsection{L'exemple d'un groupe terroriste à dénomination polémique : « Daech »}

31 Lors de l'analyse qualitative des «groupes» terroristes, il est possible de retracer la présence d'un paradigme désignationnel (Mortureux 2001 : 100), par lequel les groupes sont souvent reformulés en discours par les cohyponymes «Daech» (38 occurrences) et «État islamique » (37 occurrences). Ces deux termes sont coréférentiels et renvoient au groupe terroriste qui a été défini par le Conseil de sécurité des Nations unies (IATE : fiche 3550620).

32 Rappelons brièvement que "Daech» est le mot arabe utilisé officiellement par le Président français François Hollande et par le Quai d'Orsay depuis 2014 et que ce mot est devenu un véritable "lieu discursif» (Krieg-Planque 2006) lors de son utilisation à l'intérieur du discours présidentiel au lendemain des attentats parisiens du Bataclan en novembre 2015. Dans ce discours, où Daech est reformulé comme "armée djihadiste, armée terroriste, notre ennemi ", les tons et les termes utilisés sont empruntés à l'administration américaine de Bush (Maison 2016 : 21).

Or, si nous analysons les occurrences de ce terme dans notre corpus, nous pouvons remarquer que, dans la plupart des cas (23 occurrences), il apparaît en couple avec l'EIIL, c'est-à-dire l'acronyme français utilisé par le Conseil de sécurité de l'ONU comme forme abrégée de la dénomination «État islamique en Irak et au Levant ». D’ailleurs, dans la base de données terminologique IATE, c'est justement le couple «EIIL/Daech » qui a non seulement une fiabilité majeure (4 étoiles) ${ }^{14}$ mais qui est recommandé plutôt que le seul «Daech » qui a une fiabilité minimale (2 étoiles). Remarquons encore que dans le couple «EIIL/Daech», le désignant «Daech», qui renvoie au positionnement français, suit l'acronyme de l'« État islamique en Irak et au Levant ».

34 Malgré la recommandation de la fiche d'IATE, il reste encore quelques rares contextes où « Daech » est utilisé tout seul dans notre corpus. L'analyse de l'utilisation discursive de ce terme, démontre qu'il est présent dans les cas suivants :

1. l'énonciateur qui rédige le rapport est français : c'est le cas du rapport de Vincent Peillon;

2. les documents sont traduits en français : c'est le cas du rapport de l'autrichien Josef Weidenholzer, dont la version anglaise utilise «ISIL/Daesh» tandis que la traduction française remplace normalement cette expression par «Daech » tout court; un autre exemple est le paragraphe $17 \mathrm{du}$ rapport de la polonaise Anna Elżbieta Fotyga où la traduction française opère le même remplacement ;

3. l'éventuelle présence d'un rapporteur de langue romane, qui aurait tendance à utiliser « Daech » dans la variante graphique adaptée à sa propre langue. C'est le cas de l'avis minoritaire de l'espagnol Javier Couso, dont l'espagnol «Dáesh» est traduit en français par l'équivalent le plus harmonisé (Daech).

Toutes ces remarques suggèrent que l'utilisation d'un désignant à la place d'une autre expression / dénomination coréférentielle dépend du positionnement des énonciateurs concernés (rédacteurs ou traducteurs) et à la manière dont ils privilégient un point de vue précis par rapport à l'objet concerné. Cela est rendu encore plus évident par le fait que le terme « Daech » est également présent dans des projets des rapports en français, comme celui de Rachida Dati, mais que cette dénomination est remplacée par d'autres lors des amendements et finit finalement par disparaitre dans les versions finales de ces documents. À ce sujet, si nous comparons le projet de rapport de Rachida Dati avec la 
version approuvée du rapport français, nous voyons justement la présence du lissage qui finit par privilégier la reformulation de "Daech" par «État islamique ", ce syntagme étant la dénomination qui circule à partir de 2014, après la proclamation du califat, et qui est enregistrée comme fiable (3 étoiles) dans IATE :

(Projet de rapport) considérant que plus de 5000 citoyens européens ont rejoint des organisations terroristes et notamment les rangs de Daech en Iraq et en Syrie (Version finale) considérant que plus de 5000 citoyens européens ont rejoint des organisations terroristes et d'autres formations militaires, notamment les rangs du groupe "État islamique ", du Front al-Nosra et d'autres organisations en dehors de l'Union européenne, en particulier dans les régions du MoyenOrient et de l'Afrique du Nord

$$
\begin{aligned}
& \text { l'égard du référent désigné, d'autant plus que l'utilisation de cette expression dans les } \\
& \text { discours du PE en français s'accompagne la plupart du temps d'un commentaire } \\
& \text { autonymique plus ou moins explicité. }
\end{aligned}
$$

\section{Rachida Dati en français et en anglais :}

(Version française) considérant que plus de 5000 citoyens européens ont rejoint des organisations terroristes et d'autres formations militaires, notamment les rangs du groupe « État islamique "

(Version anglaise) whereas more than 5000 European citizens have joined terrorist organisations and other military formations, particularly ISIS (Da'esh)

Rapporteure : Rachida Dati (3/11/2015)

La comparaison montre que c'est bien dans la version française que la modalité autonymique et l'ajout du nom collectif (Ncoll) «groupe » s'inscrivent dans la matérialité discursive. L'impossibilité de garder le seul «Daech» pendant la procédure des amendements est donc partiellement compensée par la reformulation française du rapport final qui permet tout à la fois de :

1) mettre à distance le syntagme "État islamique » par la modalité autonymique marquée par les guillemets, ce qui renverrait à la non-coïncidence entre les sujets concernés, dans ce cas l'énonciateur et l'autre absent (groupe qui s'appelle / qu'on appelle / soi-disant État islamique);

2) produire un court-circuit sémantique par l'ajout du Ncoll " groupe », qui normalement serait suivi par les membres du collectif ("groupe de $+\mathrm{X}$ ») ou bien par un adjectif ("groupe + Adj.»), mais qui ici est suivi d'un autre nom, ce qui lui permet de fonctionner 
en attribut à valeur prédicative par rapport au syntagme nominal (l'État islamique est un groupe). C'est justement la coréférence entre " groupe » et «État » qui crée le paradoxe sémantique car l'«État» n'est pas un groupe d'un point de vue intensionnel et que ce n'est que par extension qu'on peut éventuellement lui attribuer cette prédication. À cet égard, les guillemets renvoient justement à la mise à distance de la prédication en question et donc à la non-coïncidence entre le mot et la chose (ce n'est pas vraiment un État mais c'est un groupe), ce qui renforce, par conséquent, la non-coïncidence entre les sujets concernés (groupe qui s'appelle / qu'on appelle / soi-disant État islamique mais qui pour le rapporteur / l'UE n'est qu'un groupe).

On retrouve le même décalage discursif (amplification du dit par l'ajout du Ncoll) et lexical (dénomination choisie) dans d'autres versions françaises des rapports, comme par exemple celle de la Polonaise Anna Elżbieta Fotyga du 14 octobre de 2016. Voici ce que nous montre la comparaison des versions polonaise et anglaise du rapport, l'une de ces deux langues étant sans doute choisie pour la rédaction du rapport, avec la version française de ce même document :

(Version anglaise) Underlines that Islamist terrorist organisations, especially ISIS

and Al-Qaeda,

(Version polonaise) podkreśla, że islamskie organizacje terrorystyczne, zwłaszcza

ISIS i Al-Kaida,

(Version française) souligne que les organisations terroristes islamistes,

notamment le groupe « État islamique » (EI) et Al-Qaida, (...)

Rapporteure : Anna Elżbieta Fotyga (14/10/2016)

Dans ce cas, nous constatons que si le français privilégie toujours la configuration "groupe + 'État islamique'", les autres versions linguistiques optent pour «ISIS » tout court.

Plus généralement, nous remarquons que, parmi les 37 occurrences françaises du syntagme «État islamique » qui sont présentes dans notre corpus, la configuration la plus utilisée est justement " groupe 'État islamique'« (18 fois); sinon, c'est le marqueur « soidisant » qui renvoie à la non-coïncidence entre les énonciateurs concernés (Féron, Coltier $2013: 276)^{16}$ et/ou entre le mot et la chose, quand on ajoute "groupe », comme dans les cas suivants :

[le PE] estime qu'une politique étrangère et de sécurité européenne plus unifiée et, dès lors, plus efficace peut contribuer de manière décisive à réduire l'intensité des affrontements armés en Iraq et en Syrie et à éliminer le soi-disant État islamique Rapporteur : Ioan Mircea Paşcu (3/11/2016)

[le PE] invite une nouvelle fois les membres du Conseil de sécurité des Nations unies à soutenir la saisine de la CPI par le Conseil de sécurité afin d'enquêter sur les violations commises en Iraq et en Syrie par le groupe soi-disant État islamique (Daech) contre les chrétiens (chaldéens, syriaques et assyriens), les yézidis et d'autres minorités religieuses ou ethniques ;

Rapporteur

: Josef Weidenholzer $(28 / 11 / 2016)$

Ce n'est donc pas un hasard si, dans l'exemple rapporté dans la fiche d'IATE sur l'État islamique, nous lisons :

Nous, Français de France et de confession musulmane, tenons à exprimer avec force notre totale solidarité avec toutes les victimes de cette horde de barbares, soldats perdus d'un prétendu État islamique, et dénonçons avec la dernière énergie toutes les exactions commises au nom d'une idéologie meurtrière qui se cache derrière la religion islamique en confisquant son vocabulaire. 
49

Cette mise à distance explicite de l'objet ("prétendu») confirme le positionnement français vis-à-vis d'un regroupement de soldats, ici reformulé en véritable horde barbare, qui n'est pas reconnu comme État, mais qui se prétend tel et qui est bien loin de l'Islam traditionnel.

Pour revenir à nos rapports, remarquons encore la présence du lissage lors de la formulation de ce référent comme d'un groupe (plus rarement une organisation) tout court, l'adjectif «terroriste " n'étant utilisé que deux fois, ce qui explique que la démarche quantitative ne l'avait pas révélé. De nouveau, l'élément religieux et le terrorisme ne font pas amalgame, bien au contraire, ce qui montre la volonté explicite des députés de proposer un discours différent sur l'objet en question :

[le PE] condamne toute analyse entraînant une confusion entre Islam, d'une part, et terrorisme, insécurité et migrants, d'autre part ;

Résolution du PE du 9 juillet 2015

considérant que le terrorisme et la radicalisation mènent à de nombreux amalgames envers les religions, qui entraînent par là-même une recrudescence des crimes et des discours de haine motivés par le racisme, la xénophobie ou l'intolérance à l'égard d'une opinion, d'une croyance ou d'une religion ; qu'il est essentiel de rappeler que le dévoiement de la religion, et non la religion en tant que telle, est une des causes de la radicalisation;

Rapporteure : Rachida Dati (03/11/2015)

51 C'est sans doute pour éviter toute indistinction entre l'islamisme et l'Islam que l'on se positionne par le lissage, mais c'est aussi et surtout pour proposer un référent contractuel qui n'est pas identique au référent circulant dans l'hyperlangue (Auroux 1997) nationale et internationale.

Cela dit, il est utile de constater que, malgré le lissage institutionnel, l'analyse restitue les traces de la présence du point de vue politique français, qui ressort finalement lors de la rédaction et /ou de la traduction des documents concernés.

\section{Conclusions} internationales, le concept de terrorisme qui circule dans le discours du PE renvoie à quelque chose de différent et devient un terme qui n'équivaut donc pas au mot courant ${ }^{17}$. Le préconstruit langagier caractérisant les hyperlangues citées et qui est étroitement lié à la religion, notamment à l'Islam via l'islamisme, est ce qui motive sans doute le positionnement des députés contre tout amalgame et surtout l'effort d'entraver l'hétérogénéité diaphasique lors d'un processus de lissage qui caractérise le discours institutionnel des rapports d'initiative du PE et, à plus forte raison, le discours législatif « instituant » des rapports législatifs ${ }^{18}$.

Bien que le référent désigné par le terme soit contractuel, il reste flou et ne peut pas se confondre avec les référents sociaux qui circulent plus amplement dans les discours extérieurs au dit des institutions européennes. Il semble désigner plutôt un objet à valeur praxématique (Siblot 1997) dans la mesure où il déclenche un programme de sens spécifique (de lutte et de prévention) beaucoup plus qu'une représentation sociale large. Il s'agit d'une menace à la libre circulation de l'information (voir la lutte à la propagande terroriste) et des personnes (voir la question des "combattants étrangers ", qui est d'ailleurs liée étroitement au voyage, tout comme les données des dossiers passagers du 
PNR du rapport cité de Timothy Kirkhope), ainsi qu'à la sécurité des citoyens. C'est donc ce qui permet de déclencher une riposte collective qui ressoude les liens de l'Union en son intérieur et avec ses voisins au point de devenir l'un des mots-arguments validant sa réforme institutionnelle interne. D'où son utilisation dans le cadre des commissions parlementaires qui concernent les affaires intérieures, extérieures et constitutionnelles. Dans cette représentation générique, quelques catégories d'actant peuvent se profiler, notamment des groupes spécifiques comme le prétendu État islamique, désignant qui permet de retracer la présence d'une représentation décalée, à savoir les traces d'un positionnement précis, celui des Français, et qui renvoie à l'effort de légitimer la représentation du «terrorisme » en tant que référent social ${ }^{19}$ tel qu'il est construit dans le discours politique en France.

Par conséquent, si la représentation du terrorisme dans le discours du PE reste liée au référent contractuel de l'eurojargon communautaire, qui tend à lisser la surface énonciative (positionnements, interférences...) tout en restant perméable à l'influence du discours onusien, on peut tout de même détecter les traces résiduelles d'un référent social "autre», une représentation alternative qui laisse des traces du positionnement national, dès qu'on considère les eurojargons spécifiques, tel l'eurojargon français. C'est ce qui mérite sans doute des approfondissements, notamment en comparaison avec les autres eurojargons nationaux.

\section{BIBLIOGRAPHIE}

\section{Bibliographie générale ${ }^{20}$}

Anthony L. 2018, AntConc <http://www.laurenceanthony.net/software.html>

Auroux S. 1997. «La réalité de l'hyperlangue », Langages, n 127, p. 110-121.

<http://www.persee.fr/doc/lgge_0458-726x_1997_num_31_127_2128>

Balibar R. 1993. Le colinguisme. Paris : PUF.

Charaudeau P. 2007. « Les stéréotypes, c'est bien. Les imaginaires, c'est mieux ». In : Henri Boyer (éd). Stéréotypage, stéréotypes : fonctionnements ordinaires et mises en scène. Paris :

L'Harmattan.

<http://www.patrick-charaudeau.com/Les-stereotypes-c-est-bien-Les,98.html\#nh9>

Charaudeau P., Maingueneau D. 2002. Dictionnaire d'Analyse du discours. Paris : Seuil.

Cussò R., Gobin C. (éds.) 2008. « Du discours politique au discours expert ». Mots. Le langage du politique, $\mathrm{n}^{\circ} 88$.

Dufour F., Rosier L. (éds) 2012. « Analyse du discours à la française : continuité et reconfigurations ", Langages et Société, n 140.

Féron C., Coltier D. 2013. « Étude de cas : soi-disant », Pratiques. Linguistique, littérature, didactique, $\mathrm{n}^{\circ} 159-160$, p. 275-294. 
InterActive Terminology for Europe - IATE <http://iate.europa.eu/>

ISO 2009. Norme internationale 704. Travail terminologique - Principes et méthodes. Genève.

Krieg-Planque A. 2006. « 'Formules' et 'lieux discursifs' : propositions pour l'analyse du discours politique (Entretien avec Alice Krieg-Planque, par Philippe Schepens) ». Semen, $n^{\circ} 21$. <https:// journals.openedition.org/semen/1938>

Krieg-Planque A. 2009. La notion de «formule » en analyse du discours. Cadre théorique et méthodologique. Besançon : Presses Universitaires de Franche-Compté.

Krieg-Planque A. 2012. Analyser les discours institutionnels. Paris : Armand Colin.

Krieg-Planque A., Oger C. 2010. « Discours institutionnels. Perspectives pour les Sciences de la Communication », Mots. Les langages du politique, n 94, p. 91-96.

Krieg-Planque A., Oger C. 2017. « Éléments de langage ». Publictionnaire. Dictionnaire encyclopédique et critique des publics. <http://publictionnaire.huma-num.fr/notice/elementsde-langage/>

Maingueneau D. 2007. « Genres de discours et modes de généricité ». Le français d'aujourd'hui, n - 159 , p. 29-35.

Moirand S. 2007. Le discours de la presse quotidienne. Observer, analyser, comprendre. Paris : PUF.

Mortureux M.-F. 2001. La lexicologie entre langue et discours. Paris : Armand Colin.

Née Émilie (dir.) 2017. Méthodes et outils informatiques pour l'analyse des discours. Rennes : Presses Universitaires de Rennes.

Née É, Sitri F., Véniard M. 2016. « Les routines, une catégorie pour l'analyse de discours : le cas des rapports éducatifs », Lidil, $\mathrm{n}^{\circ}$ 53, p. 71-92.

Nugara S. 2011. « Féminisme et universalisme du Conseil de l'Europe : le cas de la dénomination violence domestique à l'égard des femmes, Synergies Italie, nº 7, p. 39-49.

Oger C., Ollivier-Yanniv C. 2003. « Du discours de l'institution aux discours institutionnels : vers la constitution de corpus hétérogènes ». X Colloque bilatéral franco-roumain, CIFSIC Université de Bucarest, 28 juin - 3 juillet 2003.

<https://halshs.archives-ouvertes.fr/sic_00000717/document>

Oger C., Ollivier-Yanniv C. 2006. « Conjurer le désordre discursif. Les procédés de 'lissage' dans la fabrication du discours institutionnel », Mots. Le langage du politique, $n^{\circ} 81$, p. 63-77.

Parlement européen. Résolution du Parlement européen du 11 février 2015 sur les mesures de lutte contre le terrorisme (P8_TA(2015)0032)

Parlement européen. Résolution du Parlement européen du 9 juillet 2015 sur le programme européen en matière de sécurité (P8_TA(2015)0269)

Perrot M. 2002« Mondialiser le non-sens », Revue du MAUSS, n² 20, p. 204-221. <http:// www.cairn.info/revue-du-mauss-2002-2-page-204.htm>

Raus R. 2013a. « L'eurojargon et sa variante française ». Argotica, $n^{\circ} 2$.

<http://cis01.central.ucv.ro/litere/argotica/1.\%20Argotica_Ro/files2013/Argotica_1(2)2013.pdf > Raus R. 2013b. La terminologie multilingue. La traduction des termes de l'égalité H/F dans le discours international. Bruxelles : De Boeck. 
Raus R. 2017. « Les rapports d'initiative au Parlement européen ou comment la traduction influe sur les aspects performatifs d'un genre discursif », Mots. Les langages du politique, $\mathrm{n}^{\circ} 114$, p. 95-115.

Siblot P. 1997. « Nomination et production de sens : le praxème », Langages, $\mathrm{n}^{\circ}$ 127, p. 38-55.

<http://www.persee.fr/docAsPDF/lgge_0458-726x_1997_num_31_127_2124.pdf>

Tropes 2014. Version 8.4. <https://www.tropes.fr>, logiciel développé par Pierre Molette et Agnès Landré sur la base des travaux de Rodolphe Ghiglione.

\section{Bibliographie du corpus}

Bergeron J. (06/07/2017). Rapport sur les systèmes pénitentiaires et les conditions dans les prisons. PE601.203v02-00

Dati R. (03/11/2015). Rapport sur la prévention de la radicalisation et du recrutement de citoyens de l'Union par des organisations terroristes. PE551.967v02-00

Fotyga A. E. (14/10/2016). Rapport sur la communication stratégique de l'Union visant à contrer la propagande dirigée contre elle par des tiers. PE582.060v03.00

Gahler M. (13/11/2017). Rapport annuel sur la mise en œuvre de la politique de sécurité et de défense commune. PE609.294v02.00

Hohlmeier M. (12/07/2016). Rapport sur la proposition de directive du Parlement européen et du Conseil relative à la lutte contre le terrorisme et remplaçant la décision-cadre 2002/475/JAI du Conseil relative à la lutte contre le terrorisme. PE577.046v02-00

Kirkhope T. (07/09/2015). Rapport la proposition de directive du Parlement européen et du Conseil relative à l'utilisation des données des dossiers passagers pour la prévention et la détection des infractions terroristes et des formes graves de criminalité. PE 549.223v03-00 Paet U. (31/10/2016). Rapport sur l'Union européenne de la défense. PE584.127v02.00 Panzeri P. A. (20/02/2015). Rapport concernant le rapport annuel 2013 sur les droits de l'homme et la démocratie dans le monde et la politique de l'Union européenne en la matière. PE541.530v03-00.

Paşcu I. M. Rapport sur la mise en œuvre de la politique de sécurité et de défense commune (selon le rapport annuel du Conseil au Parlement européen sur la politique estrangère et de sécurité commune). PE582.127v02.00

Peillon V. (18/06/2015). Rapport sur les défis en matière de sécurité au Moyen-Orient et en Afrique du Nord et les perspectives de stabilité politique. PE 549.275v02-00

Väyrynen P. (21/10/205). Rapport sur le rôle de l'Union européenne au sein des Nations unies Comment mieux remplir les objectifs de l'Union en matière de politique étrangère. PE560.600v03-00.

Verhofstadt G. (20/12/2016). Rapport sur les évolutions et adaptations possibles de la structure institutionnelle actuelle de l'Union européenne. PE585.741v02.00

Weidenholzer J. (28/11/2016). Rapport concernant le rapport annuel sur les droits de l'homme et la démocratie dans le monde, et la politique de l'Union pour 2015. PE587.429v02-00 


\section{NOTES}

1. Nous tenons à remercier les référés pour leur relecture attentive qui nous a permis d'éclairer et d'aller plus loin dans notre propos.

2. Dans les discours parlementaires, il serait plus convenable de parler des termes parce que la présence de procédures de normalisation mises en œuvre favorise justement la création de termes et de fiches terminologiques d'après les critères onomasiologiques de la terminologie traditionnelle. Cependant, nous avons préféré ne pas opposer «terme " à "mots" et avons normalement utilisé ce dernier pour renvoyer plus en général à tout élément lexical / signe verbal.

3. Pour Charaudeau (2007: 85) : «les imaginaires sont engendrés par les discours qui circulent dans les groupes sociaux, s'organisant en systèmes de pensée cohérents créateur de valeurs, jouant le rôle de justification de l'action sociale et se déposant dans la mémoire collective ».

4. L'expression désigne «le jargon technique utilisé au niveau communautaire par les institutions européennes et qui, de par son opacité, finit par être accessible aux seuls spécialistes plutôt qu'aux citoyens européens" (Raus 2013a: 384). Précisons qu'il faut différencier «l'eurojargon [entendu] comme langue transnationale, qui se caractérise par un espace de communication très diversifié et par des contacts linguistiques permettant la circulation autant $\mathrm{du}$ lexique que des structures phrastiques, et les variantes nationales de l'eurojargon qui sont concrètement utilisées par les rédacteurs et les traducteurs de l'UE » (ibidem : 386-387).

5. Cette base est disponible à l'adresse <http://iate.europa.eu/>. Un système de notation par étoiles (de 1 à 4) permet d'afficher la fiabilité des termes présents dans les fiches.

6. La "polyphonie» doit être entendue ici comme renvoyant aux voix hétérogènes des représentants des 24 États membres.

7. Le mot «terreur " n'apparaît que deux fois dans le corpus et n'est donc pas utile à des fins d'analyse.

8. À la place de terroriste, nous signalons la présence d'un terme qui est en fait une périphrase, ce qui est plutôt inhabituel dans le domaine de la terminologie prescriptive, et qui a une fiabilité minimale : il s'agit de « Personne expressément identifiée comme terroriste internationale ».

9. Dans notre corpus, «lutte antiterroriste» est présente deux fois seulement et «lutte antiterroriste » est un hapax.

10. Ces tendances caractérisent toutes les versions linguistiques des documents, comme l'ont récemment montré les travaux de Laura Mori et de son «observatoire de l'eurolecte» à l'Université des Études internationales de Rome.

11. L'expression "prévention et détection des infractions terroristes" est la seule qui soit utilisée par rapport au terrorisme (15 occurrences).

12. Dans tous les extraits cités, les caractères gras sont les nôtres. Le sujet n'est explicité qu'au début du rapport, avant les visas, les considérant et le dispositif, comme le prévoient les guides de rédaction de ce type de documents.

13. Ce n'est donc pas un hasard si dans IATE la fiche qui concerne le «djihad islamique » est indiquée non seulement comme peu fiable mais aussi qu'elle concerne le domaine de la vie politique, tout comme la fiche «djihadiste » qui a une fiabilité majeure. Le djihadiste est défini comme « les membres de réseaux et filières islamiques, et notamment les recrues d'Al Quaida, participant à la préparation ou à l'exécution d'actes terroristes ».

14. Bien que l'utilisation des acronymes puisse rendre opaque le référent désigné et violer, par conséquent, les normes de l'Organisation Internationale de Normalisation (ISO), qui justement recommande d'utiliser des termes transparents et d'éviter l'utilisation d'acronymes, le fait 
qu'EIIL soit désormais assez connu lui permet d'être suffisamment compréhensible et donc utilisable.

15. Pour l'utilisation des formulations «combattants étrangers » et «combattants terroristes étrangers » nous renvoyons à Rafaëlle Maison (2016).

16. Selon ces auteurs, « soi-disant » est un marqueur discursif qui « signale qu'il y a emprunt à un autre discours (...) et signifie une réserve du locuteur vis-à-vis de cet autre discours ».

17. Nous tenons à préciser, entre autres, le positionnement explicite vis-à-vis des médias, comme le dit clairement le rapport sur la communication stratégique de l'Union de 2016 : «souligne la nécessité de renforcer le pluralisme et l'objectivité médiatique ».

18. N'oublions pas que le discours juridique est «constituant» (Maingueneau, Cossutta 1995 : 113) et qu'à ce sujet « (l)a prétention attachée au statut de discours constituant, c'est de fonder et de n'être pas fondé. Cela ne signifie pas que les multiples autres types d'énonciations (les conversations, la presse, les documents administratifs, etc.) n'ont pas d'action sur eux ; bien au contraire, il existe une interaction continuelle entre discours constituants et non-constituants, de même qu'entre discours constituants. Mais il est dans la nature de ces derniers de dénier cette interaction ou de prétendre la soumettre à des principes » (ibidem : 112).

19. Au sens de Krieg-Planque, pour laquelle (2009: 54) une unité «signifie quelque chose pour tous ", « qui fonctionne pour tous à un moment donné » (ibidem : 95). L'auteure parle de référent social par rapport à la notion de «formule ». Nous entendons ici cette catégorie de façon plus large, toute unité stable de la langue pouvant imposer en discours des évidences référentielles à un moment donné sans déclencher pour autant des polémiques.

20. La date de la dernière consultation des sites internet est le 10 janvier 2018.

\section{RÉSUMÉS}

Dans cet article, nous nous questionnons sur la manière dont le terrorisme est représenté en tant que référent créé en discours par les commissions du Parlement européen de la législation en cours (2014-2017). Tout en adoptant une approche de lexicologie discursive, qui est également redevable de l'analyse du discours « à la française ", nous mènerons une enquête pour cerner les contours de l'utilisation discursive des mots « terrorisme » et « terroriste » ainsi que de quelques mots qui y sont liés («djihadiste, Daech, État islamique »). Bien au-delà de l'existence de référents sociaux circulant dans les discours français de l'Hexagone, le discours tissé par le Parlement européen autour du terrorisme construit et réitère un référent discursif qui se produit dans des conditions de production discursive précises et est donc influencé par les procédures de « lissage » de la matérialité discursive typique de ce type de discours institutionnel.

In this paper we intend to analyse how terrorism is represented as a discursive referent in the discourses of the current European Parliament's committees (2014-2017). We will adopt a discursive approach to the lexicon that is influenced by the French discourse analysis in order to examine the discursive occurrences of the words "terrorism" and "terrorist", as well as some related words such as "Jihadist", "Daesh" and/or "Islamic state". We will show that the European Parliament's discourse about terrorism refers to a contractual object that is different from the social referents existing in the political discourses in French and that is influenced by the "smoothing" process of the "discursive materiality" that is typical of this kind of institutional discourse. 
INDEX

Keywords : Terrorism, Representation, Contractual referent, Institutional discourse, European Parliament

Mots-clés : terrorisme, représentation, référent contractuel, discours institutionnel, Parlement européen

\section{AUTEUR}

\section{RACHELE RAUS}

Université de Turin, Italie 